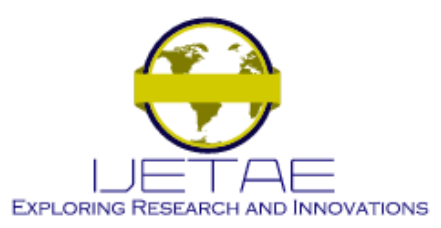

International Journal of Emerging Technology and Advanced Engineering

Website: www.ijetae.com (ISSN 2250-2459, ISO 9001:2008 Certified Journal, Volume 10, Issue 11, November 2020)

\title{
Efficiency Comparison between Robotic Manipulator Configurations for Trajectory/Path Tracing
}

\author{
Joshua Laber ${ }^{1}$, Ravindra Thamma ${ }^{2}$ \\ ${ }^{1,2}$ Robotics and Mechatronics Engineering Technology, Central Connecticut State University, USA
}

\begin{abstract}
In automation, manufacturing companies require high speed and efficiency to remain competitive in the global economy. One of the most popular ways to increase precision, speed, and accuracy is to implement industrial robotic arms. As of 2020, 2.7 million industrial robots are in operation worldwide. A robotic arm is a machine used to automatic repetitive tasks by manipulating tools or parts in the space around it. Businesses use robotic arms for many operations including pick and place, machining, welding, precision soldering, and other tasks. But with all the different types and configurations of robotic arms, the question remains: What arm would best suit the task at hand? This paper examines and compares three commonly available types of robotic arm: 5-DoF, 6-DoF, and SCARA to compare which are most efficient in tracing paths.
\end{abstract}

Keywords--Robotic Arm, Serial Manipulator, Path tracing, SCARA, Articulated, Kinematics, Forward Kinematics, Inverse Kinematics, Denavit-Hartenberg, Transformation Matrix

\section{INTRODUCTION}

Robotic arms are machines designed to perform repetitive tasks accurately and efficiently through manipulating tools and parts in its workspace. [1] Since the introduction of the first conventional robotic manipulator, the Unimate, in 1961 and its use in die-casting at the General Motors factory in Trenton, NJ, the industry around automation through the use of robotic arms had massively increased. [2] In 2020, the International Federation of Robotics announced that the number of robots operating in the world rose $12 \%$ that year to a record amount of 2.7 million. [3] The Unimate was a 5 degree of freedom robot, with two wrist axes and ran on hydraulic power technology. [2] Robotic arm technology had evolved since then to improve speed, precision, and accuracy through the use of advancing technology and different robotic configurations. Various types of robotic arm configurations include Cartesian, Cylindrical, Polar, SCARA, Delta, and Articulated. [4] The configurations covered in this paper are Articulated and SCARA. The three robotic arms that will be compared are the Fanuc LR Mate 200iC/5H, Fanuc LR Mate 200iD, and Fanuc SR-6iA.
The LR Mate 200iC/5H and LR Mate 200iD are 5-DoF and 6-DoF Articulated arms, respectively and the Fanuc SR-6iA is a SCARA arm. The forward kinematics for each robotic arm will be calculated by solving for the DenavitHartenberg parameters using the specifications listed in each robot's datasheet.

\section{COMPARING TyPes OF RoBOt CONFIGURATION}

The three robotic arms can be categorized into two categories of configuration, Articulated and SCARA.

\section{A. Articulated}

The most common form of a robotic arm is an articulated robot. An articulated robot, sometimes referred to as a jointed-arm robot, has a twisting joint to connect the arm to the body and use a series of linkages connected by rotary joints for the arm. [1] They are designed to look and to perform similar to a human arm. [5] Due to their high flexibility and agility, they are best suited for use in pick and place, welding, assembly, packaging, palletizing, material removal, and any tasks that span across planes which are not parallel. [6] They can be used in a range of environments, from dirty conditions to clean rooms with sealed rotary joints, as it is much easier to seal rotary joints over linear slides. [7] The weakness of an articulated robot is they are not as well suited for high speed tasks, since they have kinematics are more complex to calculate and they generally have a higher component mass compared to other types of robotic arm. Articulated robots are usually available as 6-DoF, but robots with higher or lower degrees of freedom are available as well. [6] The degree of freedom defines how many rotatable joints on the robot and is directly related to the number of axis of the robot. A fiveaxis robot has 5-DoF and a six-axis robot has 6-DoF. [8] An articulated robot can translate movements left/right along the $\mathrm{X}$ axis, forward/back along the $\mathrm{Y}$, up/down along the $\mathrm{Z}$ axis and rotate movements about the $\mathrm{X}$ axis (roll), $\mathrm{Y}$ axis (pitch), and $Z$ axis (yaw). [5] On a six-axis robotic arm, each axis can be compared to the structure of a human arm. The first axis is the waist, the second is the shoulder, the third is the elbow, the fourth is a wrist rotation, the fifth is a wrist bend, and the sixth are the fingertips. 


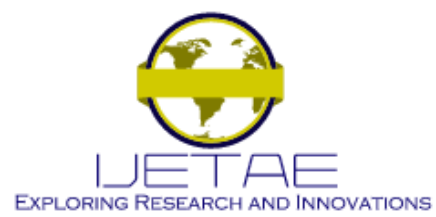

International Journal of Emerging Technology and Advanced Engineering

Website: www.ijetae.com (ISSN 2250-2459, ISO 9001:2008 Certified Journal, Volume 10, Issue 11, November 2020)

The first 3 axes make up the body and arm which control translations, while the last 3 axes act as the hand which controls orientation. [9] Compared to the six-axis robot, a five-axis robot lacks one of the joints that controls the orientation. [8]

\section{B. SCARA}

A SCARA Robot is a specific type of articulated robot which has four degrees of freedom, where three axes are rotary and the fourth is linear. SCARA stands for Selective Compliance Assembly Robot Arm, which means that arm has a rigid $\mathrm{Z}$ axis and is compliant (pliable) in $\mathrm{X}-\mathrm{Y}$ plane. [5] A SCARA arm can also be comparable to a human arm, like the revolute articulated robots. The first axis is similar to a shoulder, the second axis is an elbow, and the fourth axis rotates like a wrist. The third axis is a prismatic joint that moves rigidly in the $\mathrm{Z}$ direction. As the SCARA is a 4DoF robot, it can move freely in the $\mathrm{X}, \mathrm{Y}, \mathrm{Z}$ cartesian directions, but can only rotate in the yaw direction. This movement system is very applicable to packaging, assembly, and pick-and-place operations. [10] SCARA robots generally have a cylindrical workspace. The volume workspace can be calculated by totaling the lengths of the shoulder and elbow to determine the diameter and obtaining the $\mathrm{Z}$ stoke for the depth. One weakness of the SCARA robotic arm is as it extends outward, the arm acts like a lever which increases the amount of torque required at the motors of the joints to carry the load, which limits the robot's reach. [5] The major advantage to SCARA robots is they can have very quick cycle times in suitable applications. They are also lightweight and have compact bases which occupy little space. [6]

\section{Hypothesis}

Robots with higher degrees of freedom have increased flexibility and ability to maneuver objects compared to lower degree of freedom robots, but at the cost of speed and price per unit. Therefore, in most situations, the 4-DoF SCARA would be faster than the 5-DoF Articulated robot, which would be faster than the 6-DoF Articulated Robot. [11]

\section{CAlculations}

To understand each robot's motion, the coordinate frame system must be drawn. The forward kinematics can then be calculated as a set of parameters based on the robot's specifications and the diagrams.

\section{A. Kinematics and Coordinate Frame System Design}

The kinematics of a robot relates the position of the joints to its position in the real world.
A robot can be broken down into a set of links connected by a set of joints. There are three conventional classifications that most joints fall under: revolute, prismatic, and spherical. A revolute joint is a joint where the links revolve about one degree of rotation. A prismatic joint is a joint where the links slide linearly about one degree of translation. A spherical joint can generally be thought as two revolute joints which allows the links to rotate about a point. All three robots are defined as serial manipulators, meaning that the links follow a single chain, with no branching links. [12] The configuration of the robot can be described as the set of joint coordinates with respect to a reference frame. The configuration space is the range of all possible configurations the robot can attain, given the joint limits. [13] The workspace of a robot is the set of positions and orientations of the end-effector, also known as the end-of-arm-tool (EOAT). [12]

TABLE I.

COMPARING ROBOT SPECIFICATIONS $[18,19,20]$

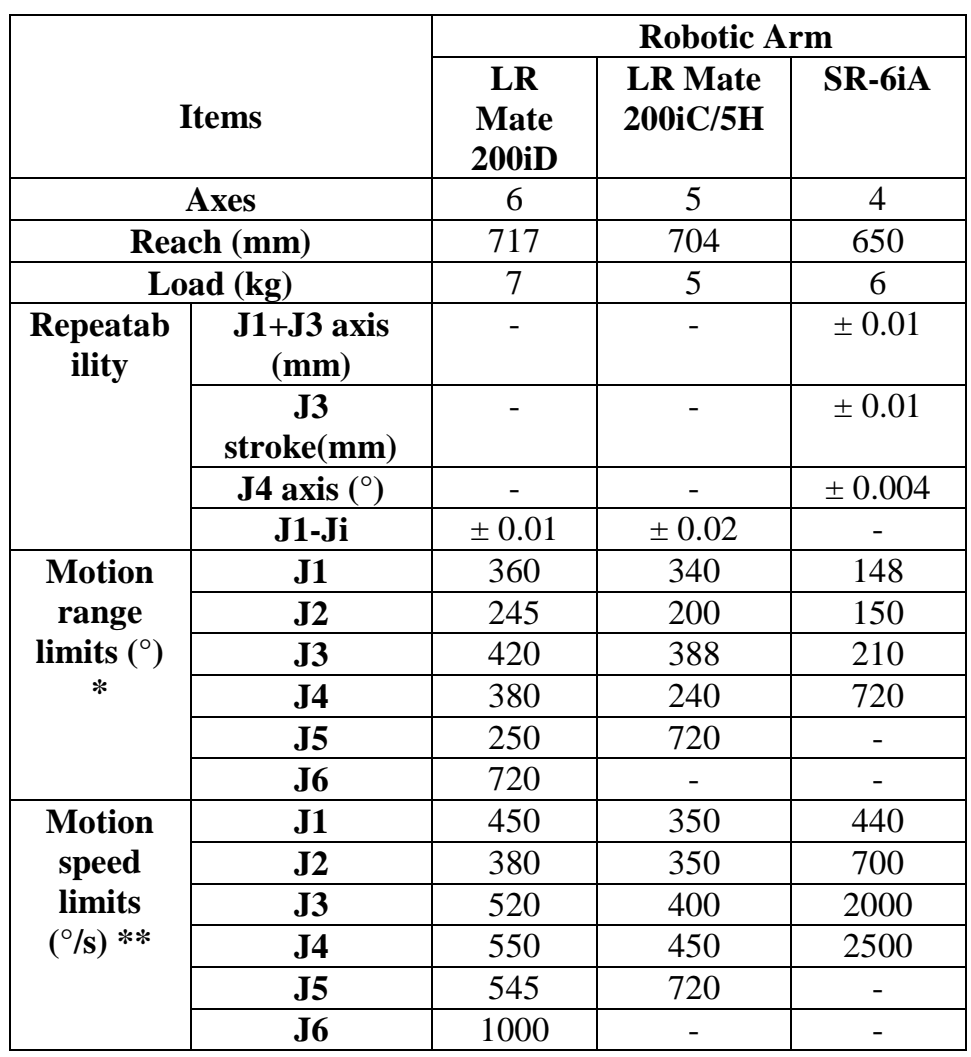

*The unit of motion range for the Fanuc SR-6iA joint J3 is $\mathrm{mm}$.

**The unit of motion speed for the Fanuc SR-6iA joint J3 is $\mathrm{mm} / \mathrm{s}$. 


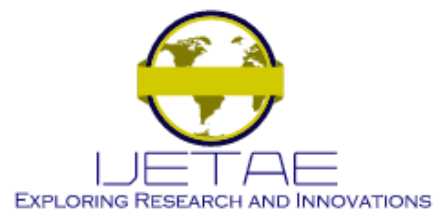

International Journal of Emerging Technology and Advanced Engineering

Website: www.ijetae.com (ISSN 2250-2459, ISO 9001:2008 Certified Journal, Volume 10, Issue 11, November 2020)

\section{B. Forward Kinematics}

Forward kinematics is the calculation of the cartesian position of the end-effector, provided the positions of the robot's joint parameters. [14] The first step in calculating the kinematics is to draw the robot as a set of coordinate frames. A serial link manipulator with $\mathrm{n}$ joints has $n+1$ links, with the joints numbered in order of base to endeffector from 1 to $n$ and the links from 0 to $n$. This means the link $i$ is affected by the actuation of joint $i$. [15] As each joint has a coordinate frame, a transformation matrix $T$ can be computed for each link. Therefore, the transformation from the base frame to the end effector can be shown as:

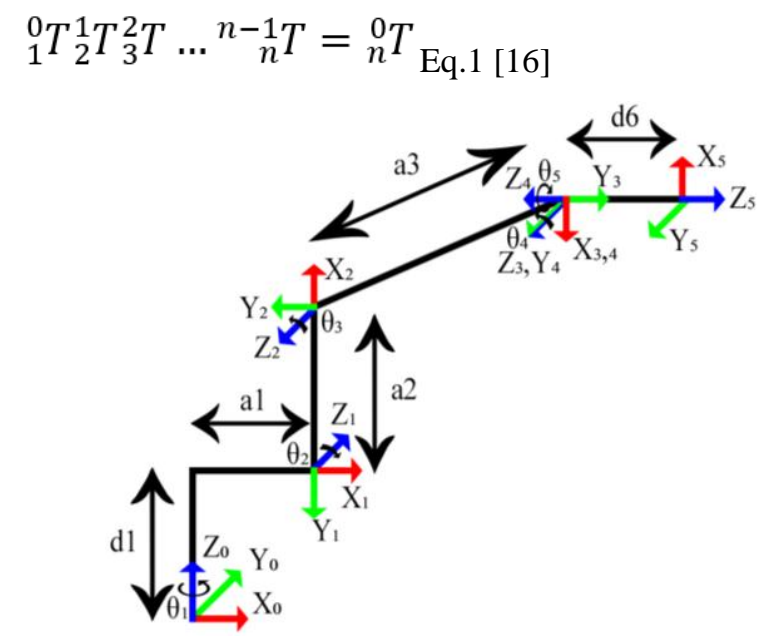

FIGURE 1. FANUC LR MATE 200IC/5H COORDINATE FRAME SYSTEM

TABLE II.

FANUC LR MATE 200iC/5H D-H PARAMETERS

\begin{tabular}{|c|c|c|c|c|c|}
\hline \multicolumn{7}{|c|}{ Fanuc LR Mate 200iC/5H } \\
\hline Joint & d & Theta & a & Alpha & Type \\
\hline 1 & 330 & $\theta 1$ & 75 & $-\pi / 2$ & Revolute \\
\hline 2 & 0 & $\theta 2$ & 300 & $\pi$ & Revolute \\
\hline 3 & 0 & $\theta 3$ & 328.671 & $\pi / 2$ & Revolute \\
\hline 4 & 0 & $\theta 4$ & 0 & $-\pi / 2$ & Revolute \\
\hline 5 & -80 & $\theta 5$ & 0 & $\pi / 2$ & Revolute \\
\hline
\end{tabular}

The method used to compute the forward kinematics of each of the three robots is the Denavit-Hartenberg (D-H) convention. [17] The notation allows the robots to be converted to a set of transformation matrices, which lets positions of the joints be easily translated to cartesian coordinates by establishing a set of rules on assigning frames.
The D-H convention assigns a set of four parameters to each frame: link length $\alpha_{i}$, link twist $\alpha_{i}$, link offset $d_{i}$, and the joint angle $\theta_{i}$. The link length $a_{i}$ is the offset distance between $z_{n-1}$ and $z_{n}$ measured along $x_{n}$ axis. The link twist $\alpha_{i}$, is the angle between $z_{n-1}$ and $z_{n}$ measured normal to $x_{n}$ axis. The link offset $d_{i}$ is the distance from the origin frame to $x_{n}$, measured along the $z_{n-1}$ axis. The joint angle $\theta_{i}$ is the angle between $x_{n-1}$ and $x_{n}$ measured normal to the $x_{n-1}$ axis. The link twist and joint angle are positive counterclockwise. The relation between the variables and the transformation matrix is:

$$
{ }_{n}^{n-1} T=\operatorname{Rot}_{z, \theta_{i}} \operatorname{Trans}_{z, d_{i}} \operatorname{Trans}_{x, a_{i}} \operatorname{Rot}_{x, \alpha_{i}}=\left[\begin{array}{cccc}
c_{\theta_{i}} & -s_{\theta_{i}} c_{\alpha_{i}} & s_{\theta_{i}} c_{\alpha_{i}} & a_{i} c_{\theta_{i}} \\
s_{\theta_{i}} & c_{\theta_{i}} c_{\alpha_{i}} & -c_{\theta_{i}} s_{\alpha_{i}} & a_{i} s_{\theta_{i}} \\
0 & s_{\alpha_{i}} & c_{\alpha_{i}} & d_{i} \\
0 & 0 & 0 & 1
\end{array}\right]
$$

Eq. 2 [15]

Where $c_{\theta_{i}}$ is $\cos \theta_{i}, s_{\theta_{i}}$ is $\sin \theta_{i}, c_{\alpha_{i}}$ is $\cos \alpha_{i}$, and $s_{\alpha_{i}}$ is $\sin \alpha_{i}$. The transformation matrix is calculated for each link where the results are input into equation 1 to obtain the total transformation from base to end effector.

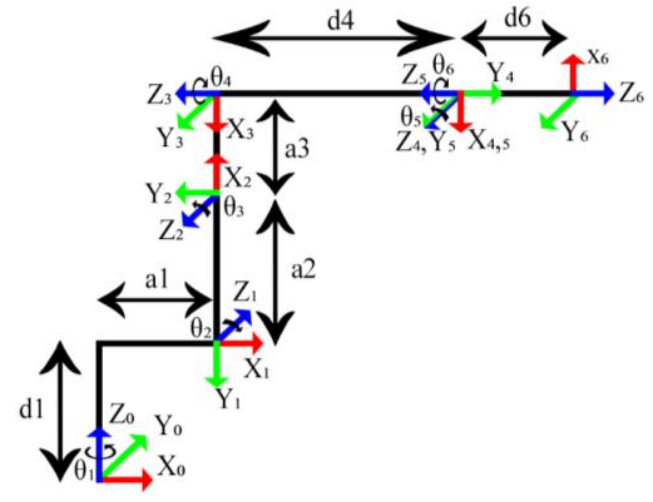

FIGURE 2. FANUC LR MATE 200ID COORDINATE FRAME SYSTEM

TABLE III.

FANUC LR MATE 200iD D-H PARAMETERS

\begin{tabular}{|c|c|c|c|c|c|}
\hline \multicolumn{7}{|c|}{ Fanuc LR Mate 200iD } \\
\hline Joint & d & Theta & a & Alpha & Type \\
\hline 1 & 330 & $\theta 1$ & 50 & $-\pi / 2$ & Revolute \\
\hline 2 & 0 & $\theta 2$ & 330 & $\pi$ & Revolute \\
\hline 3 & 0 & $\theta 3$ & -35 & $\pi / 2$ & Revolute \\
\hline 4 & -335 & $\theta 4$ & 0 & $-\pi / 2$ & Revolute \\
\hline 5 & 0 & $\theta 5$ & 0 & $\pi / 2$ & Revolute \\
\hline 6 & -80 & $\theta 6$ & 0 & $\pi$ & Revolute \\
\hline
\end{tabular}




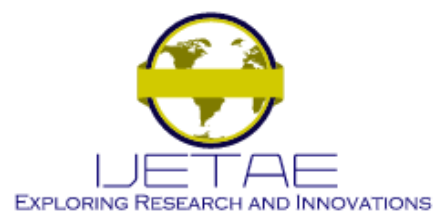

International Journal of Emerging Technology and Advanced Engineering Website: www.ijetae.com (ISSN 2250-2459, ISO 9001:2008 Certified Journal, Volume 10, Issue 11, November 2020)

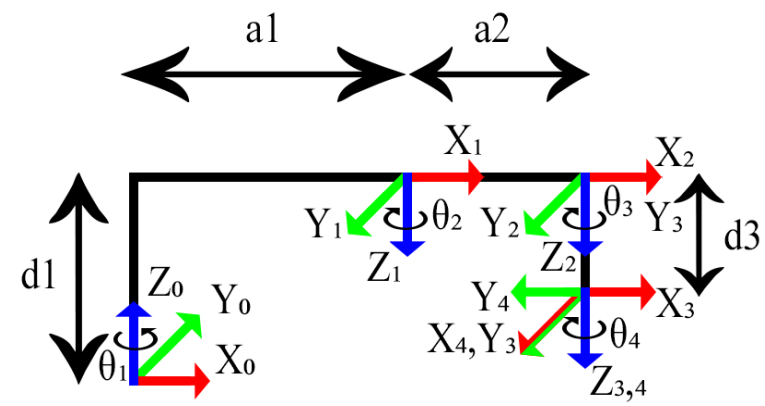

FIGURE 3. FANUC SR-6IA COORDINATE FRAME SYSTEM

\section{SYNTHESIS}

Each coordinate frame system was synthesized by rotating and translating a cartesian $\mathrm{X}, \mathrm{Y}, \mathrm{Z}$ orientation frame to follow the motion of each joint and axis. Lengths of $d$ and $a$ were obtained from the robots' datasheet. The coordinate frame system, Fig 2, and D-H parameters, Table III, for the Fanuc LR Mate 200iD were adapted from a paper by Abderrahmane et al, 2014 on the study of singularities for a six-axis robot. [21] Joint 3 of the Fanuc LR Mate $200 \mathrm{iC} / 5 \mathrm{H}$ was calculated as the hypotenuse of $a_{3}$ and $d_{4}$ on the 6-DoF version of the robot, which is similar to the Fanuc LR Mate 200iD, as the joint that would be $\theta_{4}$ is fixed on the 5-DoF version. The variable axes are $\theta_{1}, \theta_{2}$, $\theta_{3}, \theta_{4}$, and $\theta_{5}$ for the LR Mate $200 \mathrm{iC} / 5 \mathrm{H}$ and $\theta_{1}, \theta_{2}, \theta_{3}, \theta_{4}$, $\theta_{5}$ and $\theta_{6}$ for the 200iD. The coordinate frame system, Fig 3, and D-H parameters, Table IV, for the Fanuc SR-6ia were adapted from a paper by Ferrari, 2015 on controlling a SCARA Robot. [22] The variable axes are $\theta_{1}, \theta_{2}, d_{3} \theta_{4}$ for the SR-6iA.

TABLE IV.

FANUC SR-6iA D-H PARAMETERS

\begin{tabular}{|c|c|c|c|c|c|}
\hline \multicolumn{7}{|c|}{ Fanuc SR-6iA } \\
\hline Joint & d & Theta & a & Alpha & Type \\
\hline 1 & 160 & $\theta 1$ & 350 & $\pi$ & Revolute \\
\hline 2 & 0 & $\theta 2$ & 300 & 0 & Revolute \\
\hline 3 & $\mathrm{~d} 3$ & 0 & 0 & 0 & Prismatic \\
\hline 4 & 0 & $\theta 4$ & 0 & 0 & Revolute \\
\hline
\end{tabular}

\section{CONCLUSION}

The robotic manipulator configurations covered in this paper were articulated and SCARA. The examples of articulated robotic arm examined were the Fanuc LR Mate 200iC/5H and Fanuc LR Mate 200iD and the example of SCARA provided was the FANUC SR-6iA.
The advantages and weaknesses were compared between each configuration in the way of efficiency as defined by speed and agility. Robots with lower degrees of freedom would likely perform tasks faster than high DoF robots, at the cost of maneuverability. An analysis of forward kinematics was performed to create the coordinate frame system for each robot. The coordinate frames were used to generate the Denavit-Hartenberg parameters so that the forward kinematics could be easily calculated. The data and knowledge revealed can be used to model theoretical, and possibly real-world, scenarios the robots could be utilized for.

\section{RECOMMENDATION}

A future study on the kinematics of the each of the configurations should be conducted on the inverse kinematics, trajectory planning, and speed and acceleration management to understand which design is most efficient for the purpose of path planning.

\section{REFERENCES}

[1] What Is A Robot Manipulator?" RobotWorx, www.robots.com/faq/what-is-a-robot-manipulator. Accessed 13 Nov. 2020.

[2] "The First Industrial Robot: Why It Failed" Robotics Business Review, 13 Oct. 2012, www.roboticsbusinessreview.com/manufacturing/the_first_industrial _robot_why_it_failed/. Accessed 13 Nov. 2020.

[3] "IFR presents World Robotics Report 2020" International Federation of Robotics, 24 Sept. 2020, https://ifr.org/ifr-pressreleases/news/record-2.7-million-robots-work-in-factories-aroundthe-globe. Accessed 12 Nov. 2020.

[4] "What Are the Main Types Of Robots?" RobotWorx, www.robots.com/faq/what-are-the-main-types-of-robots. Accessed 13 Nov. 2020.

[5] "What Are the Types of Industrial Robots" elliTek, 24 Sept. 2020, https://www.ellitek.com/types-of-industrial-robots. Accessed 12 Nov. 2020.

[6] "4 Types of Robots Every Manufacturer Should Know" NIST, 11 July 2019, www.nist.gov/blogs/manufacturing-innovation-blog/4types-robots-every-manufacturer-should-know. Accessed 13 Nov. 2020.

[7] "Pros and Cons of 4 Industrial Robot Types" Keller Technology Corporation, 1 Mar. 2017, www.kellertechnology.com/blog/how-touse-4-types-of-industrial-robots-to-your-advantage/. Accessed 13 Nov. 2020.

[8] "Unraveling Degrees of Freedom and Robot Axis: What does it mean to have a multiple axis pick and place or multiple axis robot?" Motion Controls Robotics, 9 July 2013, motioncontrolsrobotics.com/unraveling-degrees-of-freedom-androbot-axis-what-does-it-mean-to-have-a-multiple-axis-pick-andplace-or-multiple-axis-robot/. Accessed 13 Nov. 2020. 


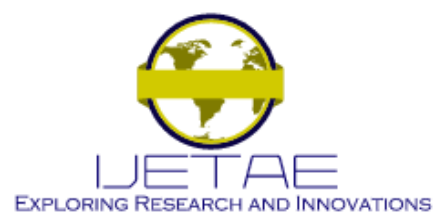

International Journal of Emerging Technology and Advanced Engineering Website: www.ijetae.com (ISSN 2250-2459, ISO 9001:2008 Certified Journal, Volume 10, Issue 11, November 2020)

[9] "How Are Industrial Robots Built? A Guide on the Components and the Movement of Robot Arms| XYZ |" Kawasaki Heavy Industries, Ltd., 22 May 2018, robotics.kawasaki.com/ja1/xyz/en/1804-03/. Accessed 13 Nov. 2020

[10] "SCARA ROBOTS-Four Decades on and Still the Most Sought After Robot" Sastra Robotics, 20 Sept. 2018, sastrarobotics.com/scara-robots-the-most-sought-after-robot/. Accessed 13 Nov. 2020.

[11] "Speed vs Flexibility: 4-axis vs 6-axis robots" RoboWorx, www.robots.com/articles/speed-vs-flexibility-4-axis-vs-6-axisrobots. Accessed 13 Nov. 2020.

[12] Hauser, Kris. "Section II. MODELING" Robotic Systems (draft), http://motion.cs.illinois.edu/RoboticSystems/Kinematics.html. Accessed 12 Nov. 2020.

[13] Varshavskaya, Paulina. "Notes on Configuration Space" Comp15007: Intelligent Robotics, https://www.cs.tufts.edu/comp/150IR/hw/cspace.html. Accessed 12 Nov. 2020

[14] Constantin, Daniel, et al. "Forward Kinematic Analysis of an Industrial Robot". DEVELOPMENTS in MECHANICS and MECHANICAL ENGINEERING, 2015. pp. 90-95., Accessed 12 Nov. 2020.

[15] Spong, Mark W, et al. Robot Dynamics and Control 2nd ed., Wiley, 2004.

[16] Soyaslan, M. et al. "Modelling, Control and Simulation of a SCARA PRR-Type Robot Manipulator." Scientia Iranica 27 (2018): 330-340.

[17] J. Denavit, and R. Hartenberg: ASME Journal of Applied Mechnics Vol. 22(1955), No. 6, p. 215.

[18] "LR Mate 200iD” Fanuc Robotics, 2019, https://www.fanucamerica.com/docs/default-source/fanuc-robotdatasheets-new/datasheet-lrmate-200id.pdf. Accessed 13 Nov. 2020.

[19] "LR Mate 200iC" Fanuc Robotics, 2009, https://www.fanucamerica.com/cmsmedia/datasheets/LR\%20Mate\% 20200iC\%20Series_10.pdf. Accessed 13 Nov. 2020.

[20] "SCARA Robot SR-6iA" Fanuc Robotics, 2019, https://www.fanuc.eu/ /media/files/pdf/products/robots/flyer/mfl03156-ro\%20sr-6ia\%20introduction/flyer-scara-robot-6ia-en.pdf. Accessed 13 Nov. 2020

[21] Abderrahmane, M. \& Djuric, Ana \& Chen, W. \& Yeh, C. (2014). Study and validation of singularities for a Fanuc LR Mate 200iC robot. IEEE International Conference on Electro Information Technology. 432-437. 10.1109/EIT.2014.6871803.

[22] Ferrari, L. "Matlab-based Control of a SCARA Robot." (2014). 